\title{
Fuzzy Based Reliable Load Balanced Routing Approach for Ad hoc Sensor Networks
}

\author{
J. V. Anchitaalagammai ${ }^{1, *}$, Rajesh Verma ${ }^{2}$, M. Kavitha ${ }^{3}$, A. R. Revathi ${ }^{4}$, S. R. Preethi ${ }^{5}$ and $^{2}$ \\ Kiranmai Bellam ${ }^{6}$
}

\footnotetext{
${ }^{1}$ Department of Computer Science and Engineering, Velammal College of Engineering and Technology, Madurai, 625009, TamilNadu, India

${ }^{2}$ Department of Electrical Engineering, King Khalid University, Abha, 62529, Saudi Arabia

${ }^{3}$ Department of Electronics and Communication Engineering, K. Ramakrishnan College of Technology, Tiruchirappalli, 621112 , Tamil Nadu, India

${ }^{4}$ Department of Information Technology, SRM Valliammai Engineering College, Chennai, 603203, Tamil Nadu, India

${ }^{5}$ Department of Electronics and Communication Engineering, SRM Valliammai Engineering College, Chennai, 603203, Tamil Nadu, India

${ }^{6}$ Department of Computer Science, Prairie View A \& M University, Prairie View, TX, United States

*Corresponding Author: J. V. Anchitaalagammai. Email: anchitaalagammailphd@gmail.com

Received: 21 June 2021; Accepted: 23 July 2021
}

\begin{abstract}
Energy management and packet delivery rate are the important factors in ad hoc networks. It is the major network where nodes share the information without administration. Due to the mobility of nodes, maximum energy is spent on transmission of packets. Mostly energy is wasted on packet dropping and false route discovery. In this research work, Fuzzy Based Reliable Load Balanced Routing Approach (RLRA) is proposed to provide high energy efficiency and more network lifetime using optimal multicast route discovery mechanism. It contains three phases. In first phase, optimal multicast route discovery is initiated to resolve the link failures. In second phase, the link quality is estimated and set to threshold value to meet the requirements of high energy efficiency. In third phase, energy model is shown to obtain total energy of network after transmission of packets. A multicast routing is established Based on path reliability and fault tolerant calculation is done and integrated with multicast routing. The routes can withstand the malicious issues. Fuzzy decision model is integrated with propose protocol to decide the performance of network lifetime. The network simulation tool is used for evaluating the RLRA with existing schemes and performance of RLRA is good compared to others.
\end{abstract}

Keywords: Fuzzy model; link quality; multicast route; energy model; cluster group; node lifetime

\section{Introduction}

MANET is one of the modes popular networks and important model for delay tolerant networks where the nodes are communicating without the need of infrastructure and discontinued path exists between source

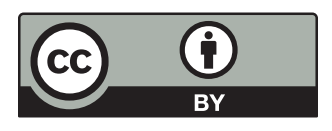

This work is licensed under a Creative Commons Attribution 4.0 International License, which permits unrestricted use, distribution, and reproduction in any medium, provided the original work is properly cited. 
and destination nodes. The information exchange among group of users was supported by the multicast delivery mechanism where source node is linked to group of sink nodes. It can be illustrated in battlefield where the commander sends message to group of soldiers without access point. In social networks and applications, single user can share audio, video, picture, and messages to group of his neighbors or friends. The packet delivery rate and energy efficiency can be improved by adopting Quality of Service [1]. Due to fluctuation of routes and mobility of nodes, energy efficiency will not be improved in ad hoc network in the presence of packet dropping. The power of the transmitter can be adjusted according to support various applications. In this research, multicast routing Based energy efficient model is introduced to attain high network lifetime by adopting various strategies and routing methodologies.

\section{Analytical Survey}

Kuo et al. [2] explored the Energy Efficiency optimization using cross layer design. The energy unit was modeled using non convex mixed integer programming. This programming was formalized by considering scheduling, energy management and routing. The problem is optimized using non convex programming. The concept of branch and bound algorithm was used to identify the issues of energy during data transmission. Riasudheen et al. [3] proposed the energy management of cloud assisted ad hoc networks to extend the network lifetime. The communication between the head nodes and mobile nodes were frequently monitored and renewed Based on performance metrics of networks i.e., overhead, route failure and batter power of node etc. The maximum energy was spent on finding and connecting the mobile nodes. The fast local route recovery mechanism was adopted to reduce the energy consumption. The intermediate nodes were grouped together to form overlay network which consumes less energy if more link failure occurred.

Zhang et al. [4] developed an offline roadside scheduling algorithm to provide a better energy consumption. The concept of on/off scheduling was to determine the slots for packet forwarding towards the sink node through the channel. The size of contention window was adjusted according to the network overload. If the density is more, the size can be limited to reduce the packet loss during packet transmission. The optimization issues were solved by the non-linear programming to reduce the energy consumption. Meanwhile the sub optimal issues were solved by the non-linear programming model. Peng et al. [5] designed an energy efficient cooperative multi input multi output transmission for increasing network lifetime of ad hoc networks. A new cooperative strategy was followed Based on random distribution methods with various number of nodes. The synchronization and issues of internal packet transmission were solved using spatial modulation technique. The information of transmission was transmitted by cluster members with supporting nodes to achieve the flexibility. The energy consumption of the cooperative strategy was estimated and compared with existing schemes and shown the better performance.

Malekshan et al. [6] proposed the concept of joint transmission scheduling and power control mechanism to improve the network performance. Due to unbounded network region and high node density, the concept of asymptotic optimal scheduling and transmission power control was determined. The basic limits of spectrum and energy model were determined to analyze the optimal scheduling. The transmission power level was determined Based on optimal solution values. The continuous transmission at each network slot was scheduled to improve the energy utilization and spectrum efficiency. Tilwari et al. [7] presented multipath aware routing Based on node mobility, remaining energy and quality of links in the network. The multiple parameters were aggregated into a single value Based on Q learning concept in order to provide an optimal path decision. The status of nodes was evaluated in the presence of path estimation and topology sensing method to obtain stable routes in the convergence network region. During the selection of stable paths between source and sink node, least residual energy and more mobility nodes were avoided to cope with route failures and network lifetime enhancement. 
Rao et al. [8] introduced the secure cryptography method integrated with energy efficient clustering method to balance security and energy in ad hoc networks. The proactive routing scheme was implemented with energy conservation scheme. The energy status and node mobility was focused in the routing hierarchy before data transmission process. The energy cost was estimated and attained Based on individual node energy estimation with the help of K-Means clustering along with Ad-hoc On Demand Distance Vector routing method. For security, the packets were protected with advanced encryption standard.

Ramya et al. [9] introduced the enhanced range routing with on demand routing to improve energy efficiency of network. Here only few nodes were participated for processing and receiving the data Based on signal strength to reduce energy consumption and overhead. An adaptive strategy was adopted to improve energy efficiency and transmission distance using enhanced range routing method. Here individual zones were particularly monitored to attain maximum network lifetime. Kanagasundaram et al. [10] proposed the multi objective ant lion optimizer to prolong the network lifetime and minimize the delay. The weight factor was calculated on shortest routes to improve the energy efficiency of individual node. The best and stable optimal path was selected to monitor the reputation metric, threat value and suspicious activity between the nodes. The degree of vulnerability was determined Based on energy conservation and misbehaving activity in the routes. The new performance metrics were introduced and tested for increasing the energy of link state routing. The multipoint relay path was selected Based on high integrity rate and maximum energy value.

Jubair et al. [11] presented a new link state routing called Bat Optimized Link State Routing (BOLSR) protocol to improve energy efficiency of network in the presence of high mobility nodes. The maximum energy was spent on route discovery and packet transmission period. The optimal paths were the solution to provide high residual energy to improve the network lifetime. Both link state routing and bat routing algorithm was used to identify the best routes Based on energy dynamic values. Fatima et al. [12] introduced the strategies for reducing energy consumption using specific energy modela and dynamic ad hoc on demand method. The power saving method was deployed to provide additional features of Wireless Local Area Network (WLAN). A multi hop relay model was taken into a consideration to improve coverage area with least energy consumption. Residual life battery monitoring model was adopted to focus on the energy value of WLAN and IEEE 802.16 standards and technologies.

Kaur et al. [13] have reviewed and presented different fault tolerant routing algorithms and protocols for ad hoc wireless networks. Various handling problems like node failures, link failures, and transmission power and energy dissipation were analyzed and solutions were given to those issues. The overall performance Based on throughput, reliability and network lifetime was recorded in the presence of fault tolerant routing schemes. Ravichandra et al. [14] presented Fault tolerant QoS Routing Protocol (FTQRP) to attain high tolerant route in the presence of mobile environment. Alternate routes are discovered if the route breaks occurs. Packets are sent through alternative routes to achieve more packet delivery ratio. Mobile nodes are randomly moving inside and outside the network. In previous work of this protocol, genetic algorithm was implemented to address routing with the help of network redundancies. In this QoS protocol, more fault tolerant rate has been improved than genetic algorithm.

Palaniappan et al. [15] introduced energy efficient QoS routing to estimate parameters of link reliability with the help of fuzzy logic technique. While adopting this approach, routing layer will be more robust. From the OSI architecture, link layer, routing and physical layer performs better. The Signal to Interference plus noise ratio can be calculated using physical layer. The neighbor status and back off time can be estimated for link layer. Data rate and data count information were calculated to decide the packet transmission status. Chaudhary et al. [16] have made an analysis on fault tolerant methodologies to route packets along the optimized paths. All the paths have threshold signal strength to achieve more energy efficiency. Route 
discovery and route maintenance are continuously monitored to avoid packet loss in the presence of link mobility.

Tavakoli et al. [17] proposed efficient fault-tolerant routing algorithm for MANET. Network fault tolerance and natural redundancy was successfully improved using this algorithm. Initially, selection $\mathrm{f}$ backup routes and nodes were chosen by predicting reputation parameters. Fault tolerant routing will be initiated once the selection of backup of nodes was over. The primary route between pair of source and destination was estimated. Nallusamy et al. [18] introduced the new reliable protocol called Mobile Agent Based Energy Efficient Reliable routing protocol to improve the link cost metrics i.e., network load and link availability. All the mobile agents are randomly deployed and packets are transferred in a hop by hop manner once it reaches the destination. From this traversal, node agents can collect the combine link cost metrics and source agents may able to select the optimal path.

Rani et al. [19] have introduced the new concept of link optimization procedure to extract the bandwidth efficiency using window channel. It was considered that previous obstacles and solutions were found to improve the energy efficiency. The optimal network path was established from source to destination by considering link cost metrics and node trust parameters. Meena et al. [20] proposed the topology transparent routing to achieve the improvement in network performance. There are three different network scenarios adopted to attain more gain. Network was kept as static and dynamic. Energy estimation was done in the last scenario. Routing steps were modified according to the energy level of mobile nodes. The concept of shortest path mechanism was established to achieve high energy efficiency. Narayanan et al. [21] presented fault tolerant routing for selecting cluster head with maximum energy efficiency. The link failure was mitigated by deploying local repair method. This method was utilized for avoiding link breakages. However, this routing suffered from excessive routing overhead and more delay.

Nandhini et al. [22] developed an effective and secured message broadcasting model to achieve better results over authentication with least energy consumption. The secure broadcast model was used to minimize overhead Based on the evaluation of confidentiality and authentication through source node. The confidence correlation measure was calculated to find the authenticated route between source and neighbor nodes. Gaur et al. [23] introduced a multi-constraints integrated link Based multicast routing protocol while considering Quality of Service (QoS) metric to improve throughput, and improve bandwidth efficiency of MANET. The link stability factor was calculated to determine the number of stable links in the network. The authors used the concept of mesh Based routing to support mesh communication while reducing link failures. The proposed RLRA contains five sections. Section 1 deals with MANET overview and need for energy efficiency. Section 2 surveys the various methods and protocols relevant to proposed approach. Section 3 discusses the proposed approach that contains network model and routing methods. Section 4 deals with simulation results and last section concludes the proposed work.

The proposed work is contributed to improve reliability with following innovations

1. The path reliability metric is calculated Based on energy, bandwidth and delay values to ensure good network lifetime.

2. Establishment of optimum energy model is to increase network energy efficiency.

3. Link availability is determined to maximize the packet delivery ratio.

4. Cluster group with network density is identified to face fault failures through the calculation of fault tolerant ratio. 


\section{Reliable Load Balanced Approach}

In this section, Reliable load Balanced approach is developed to improve energy efficiency of nodes in the network with optimal route selection in multicast route and energy efficient model. Before data transmission process, the network model is introduced to attain maximum energy.

\subsection{Network Model}

In this phase, the topology of MANET is derived as $\mathrm{T}(\mathrm{n}, \mathrm{r})$. Where $\mathrm{n}$ indicates nodes and $\mathrm{r}$ means routes. Both are connected by the devices i.e., edges. The reliability of link is expressed as $l_{r}$ and residual energy of node is expressed as $E_{r}$.

\subsection{Multicast Route Establishment Based on Path reliability}

Multicast routes are established from source to sink whereas the secured data packets are forwarded through routes. The routes are able to withstand dynamic environment, but it is difficult to identify attackers in the coverage region. Mostly routes are used as disjoint routes. The remaining energy of the node and signal strength decides the cluster heads and members which forms cluster region. Mesh Based multicast routing is implemented to improve network connectivity by sending group of messages to many sink nodes. The link reliability is determined Based on minimum packet dropping and reliable transmission. This metric is determined from the link reliability $\left(l_{r}\right)$, residual energy $\left(E_{r}\right)$, delay $(d)$ and bandwidth $(B W)$. The link reliability is estimated as,

$l_{r}=\frac{P_{d}-P_{l}}{P_{T}}-E_{w}$

where $P_{d}$ is the destination packets, $P_{l}$ is the packets loss, $P_{T}$ is the total packets and $E_{w}$ is the energy wastage on packet loss and retransmission of packets. During bandwidth estimation, the channel capacity and periods of sleep node and active node during packet transmission are considered. In energy model, initial energy is $E_{i}$ and transmission energy is $E_{t}$. The transmission energy is estimated Based on packet transmission $N_{t}$ and reception $N_{r}$ with respect to constant parameter $(\mu, v)$ and it is derived as,

$E_{t}=N_{t} * \mu+N_{r} * v$

The delay is measured from the propagation delay, processing delay and queuing delay. It is given as,

$d=d_{p}+d_{p g}+d_{q}$

The path reliability metric $\left(P_{k}\right)$ is measured as,

$P_{k}=P_{1} *\left(\frac{B W_{s}}{B W_{T}}\right)+P_{2} *\left(\frac{E_{t}}{E_{i}}\right)+P_{3} *\left(\frac{d}{T_{\max }}()_{4}\left(\frac{l_{r}}{l_{n}}\right)\right)$

where $P_{1}+P_{2}+P_{3}+P_{4}=1, T_{\max }$ is the maximum time for synchronization, $l_{n}$ is the number of links available in the network.

Fault tolerant routes are defined with less number of lost packets. Neighbor node checks the reliability of paths and hop count value and forward it to destination node. If the Sequence Number of the node is not matched, neighbor node will resend the RRREQ packets to source node. If it is matched, the message will be broadcasted to destination node. The destination node sends the reply packets containing path reliability count. $\mathrm{CH}$ chooses maximum reliability count and check the fault tolerability of the paths. The optimized fault tolerant route is discovered with having least energy nodes on active route inside the network region. Route error (RERR) message will be received if the energy of node falls below the 
threshold value. Here the maximum transmission energy is 0.879 Joules as fixed in the simulation setup. $\mathrm{CH}$ creates and chooses alternative path to the destination. Mesh routes are created Based on high path reliability metric and number of fault tolerant routes with least cost metric.

\subsection{Determination of Fault Tolerable Routes}

Once the alternative paths are setup towards the destination node, neighbor node discovers the optimal paths Based on the link quality estimation. Link quality defines that number of packets can be carried out through the links which does not cause packet losses. It is calculated by estimating the Expected Transmission Period (ETP). This period ensures no packet losses during data transmission. This metric is used to find link capacity $(l c)$ and packet size $(p s)$. This ETP value will be stored in all mobile nodes including source and destination. It is given as,

$E T P_{k}=E T C_{k} *(l c / p s)$

where ETC is the expected transmission count for packets.

Fault Tolerable Routes (FTR) can be found as the sum of metrics of link, remaining energy, hop distance, and ETP. It is estimated as,

$F T R=\left(\epsilon * r e_{k}+\varphi * \frac{1}{d_{j k}}+\gamma * \frac{1}{E T P_{k}}+p_{-}\right.$reliability $)$

where $\epsilon, \varphi, \gamma$ are the coefficients related to remaining energy, distance between two mobile nodes and expected transmission period. Source node chooses path with maximum FTR. Source node sends RRREQ message towards the next hop. Once it is confirmed, it is able to identify the presence of destination node. The flag will be set if the destination address matches with address in the header field of RRREQ. If not, the optimal route will be constructed towards the destination node.

\subsection{Cluster Network Model}

The cluster network model is illustrated to balance the load and energy. Cluster nodes are grouped together to form a cluster and select Cluster Head $(\mathrm{CH})$. In each cluster, one anchor node will be set to monitor the behaviour of nodes and paths. The routes are computed Based on quality of links, remaining energy of node and delay metric. The availability of links and delay are estimated. The epoch is defined here to identify the movement of nodes from one location to another location with constant speed and same direction. It is not same for all mobile nodes. Constant epoch length defines different epoch size. The cluster multicast routes are established from $\mathrm{CH}$ to cluster members. The reason for choosing mesh routing is to provide global connectivity and optimal solution to link failures. The failure rate and delay is very low in mesh routing. Meanwhile it also supports cluster formation and discovery process. The recovery of routes can be quickly done with the help of multicast routes. $\mathrm{CH}$ is the source node and destination cluster members are the target nodes. These nodes are the end node who will complete the data transmission process. $\mathrm{CH}$ sends bulk of messages to cluster members by creating multicast routes. The routes are created and formalized for packet forwarding Based on link quality. Before that, link availability must be tested to obtain quality.

The link availability $L_{A}\left(\tau_{C H}\right.$ to $\left.C M\right)$ is estimated between $\mathrm{CH}$ and destination cluster member if the constant epoch length persists. It is given as below,

$L_{A}\left(\tau_{C H}\right.$ to $\left.C M\right) \underset{=}{\Delta} A\left\{t_{C H}\right.$ to $t_{C M}+\tau$, avaliable $\}$ 
If velocity is changed between two epochs, the link availability can be computed as,

$L_{A}\left(\tau_{C H}\right.$ to $\left.C M\right)=L_{A}\left(\tau_{C H t}\right)+L_{A}\left(\tau_{C M}\right)$

If the mobility of nodes are independent, the exponential distribution of link availability is given as,

$L_{A}\left(\tau_{C H \text { to } C M}\right)=\left[1-E\left(\tau_{C H} \text { to } C M\right)\right]^{2}=e^{=2 \lambda \tau_{C H} \text { to } C M}$

The received signal strength indicator is used to identify the node location and node mobility inside or outside the region. The square distance between the nodes is degraded by the link quality if nodes are moved out of cluster. It is estimated Based on random variable $\alpha$ and given as,

$L_{q}(\alpha)=\frac{\alpha+\left(\tau_{C H} \text { to } C M-\alpha\right)}{\tau_{C H} \text { to } C M}$

Once the link quality is obtained, $\mathrm{CH}$ verifies the multiple link quality Based on threshold value. If links are fluctuated and low value, it will be isolated for packet forwarding.

\subsection{Data Transmission Through Multicast Routing}

In this phase, $\mathrm{CH}$ sends CHM_Req packets to all cluster members to initiate packet forwarding. Cluster members receive the request packets and replies via CHM_Rep packets to $\mathrm{CH}$. The destination cluster members are determined to begin packet forwarding. Once confirmed, $\mathrm{CH}$ sends data packets to destination CM via optimal routes. If any packet loss occurs, destination CM sends failure token message to $\mathrm{CH}$ to reinitiate packet transmission. This token message contains packet failure information due to errors. It may occur due to path failure or path overload. The residual energy is maintained among all cluster members. If any packet loss occurs, destination $\mathrm{CM}$ sends failure token message to $\mathrm{CH}$ to reinitiate packet transmission. If any error in the packet found, it will be immediately corrected using forward error correction. Cluster members are the nodes which are actively participating to elect Cluster Head $(\mathrm{CH})$. The illustration of data transmission in cluster multicast zone is shown in Fig. 1.

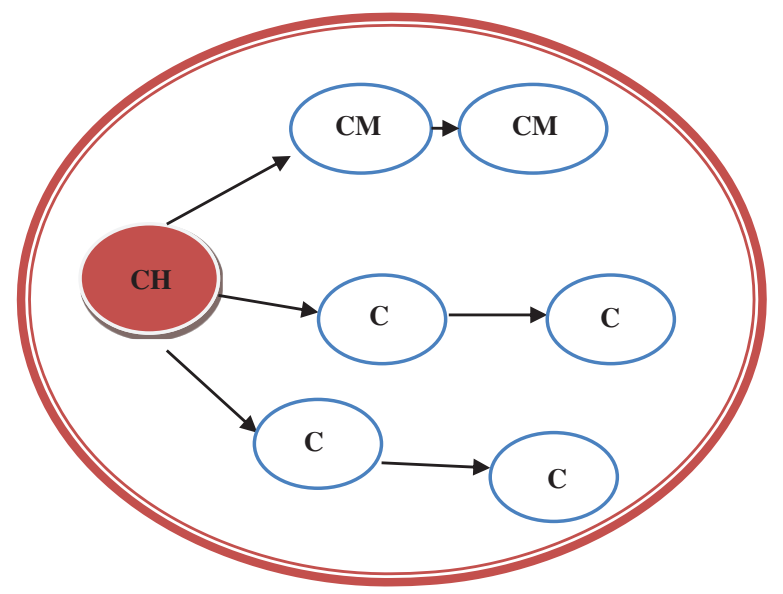

Figure 1: Data transmission in cluster multicast group

During data transmission, cluster messages are transmitted through optimal routes. It will be checked and confirmed by the $\mathrm{CH}$. 


\subsection{Optimal Energy Model}

In this phase, energy needs to be estimated during packet transmission and at the end of route maintenance phase. The The energy consumed by the packets from node to node is evaluated. The energy spent $\left(E_{S}\right)$ by $\mathrm{CH}$ and $\mathrm{CM}$ for packet forwarding is estimated as,

$E_{S}=\sum_{r=1}^{m} \tau(n r, n r+1)-\sum_{r=1}^{m} \tau_{p}(w r, w r+1)$

where $r$ indicates residual energy, $m$ means number of nodes and $n$ indicates epoch size, $w$ indicates energy wastage on packet dropping.

The residual energy is calculated as,

$r_{k}=T_{k}-E_{w}$

where $T_{k}$ is the total energy allocated to nodes and $E_{w}$ is the energy wasted on packet dropping. The transmitter $E_{t}$ energy is given as,

$E_{t}=\sum\left(E_{T}, E_{R D}\right) \times \tau$

This energy is Based on energy spent for packet transmission $E_{T}$ and route discovery process $E_{R D}$.

The receiving energy $E_{r}$ is estimated as,

$E_{r}=\sum\left(E_{R}, E_{P A}\right) \times \tau$

This energy is Based on energy spent for packet reception $E_{R}$ and packet arrival $E_{P A}$ at sink node.

The total energy $E_{T}$ is derived as

$E_{t}=E_{t}+E_{r}+E_{I}$

where $E_{I}$ is the energy spent in idle mode. The total energy of node is also estimated Based on power spent for packet forwarding from $\mathrm{CH}$ to $\mathrm{CM}$.

Total energy $=$ Total energy for cluster group/packet delivery cost.

The algorithm 1 shows illustration of RLRA to provide high energy efficiency through optimal path.

\section{Algorithm 1: RLRA algorithm}

1. Start

2. $\mathrm{CH}$ initiates route discovery process

3. $\mathrm{CM}$ joins routing and finalize destination $\mathrm{CM}$

4. Obtain the link quality

5. If threshold link quality is not met

6. \{

7. Drop the route discovery process and reinitiate

8. else

9. Initiate packet forwarding 
Algorithm 1 (continued).

10. $\}$

11. Estimate energy on transmitting and receiving section.

12. Find total energy and network lifetime

13. End

From the above algorithm, the total energy and network lifetime of sensor networks are estimated through link quality determination and packet forwarding initialization. After route maintenance process, the total energy and network lifetime will be estimated.

\section{Fuzzy decision model for Fault tolerant}

Fuzzy decision mechanism is used using Mamdani Fuzzy model. The inputs to the fuzzification are path reliability and expected transmission count. It is converted as crisp values and given to fuzzy inference engine. The values are processed and given to defuzzification. The values are converted into single output i.e., network lifetime. If both crisp values are high, network lifetime will be high. Fig. 2. shows the illustration of fuzzy decision mechanism.

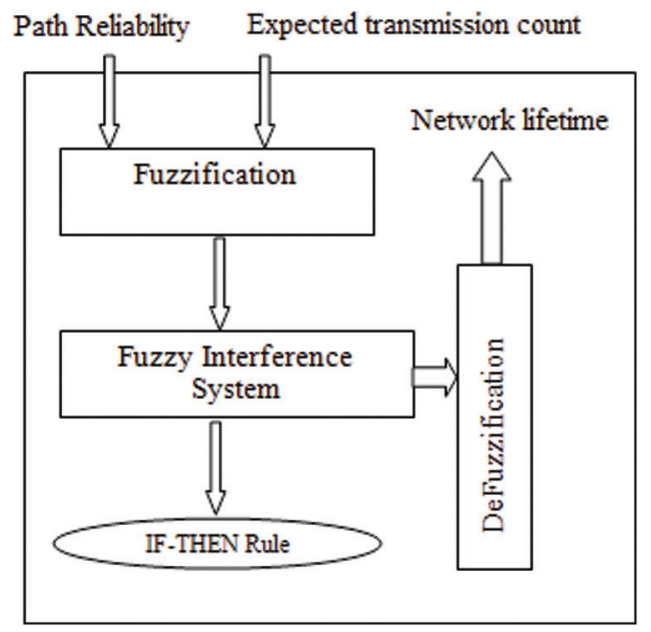

Figure 2: Fuzzy decision model

\subsection{RLRA Frame Format}

The Tab. 1 shows the illustration format of RLRA. First two field occupies 4 bytes by the identity of cluster head and cluster member as well as link quality. The link quality field is used to provide optimal solution for link failure and Route ID occupies one byte. The energy status of node is shown to know the residual energy of node after packet transmission. Cyclic redundancy check is used to detect and correct the packet error that occupies 4 bytes.

Table 1: Frame format

\begin{tabular}{lllll}
\hline $\mathrm{CH}$ and $\mathrm{CM}$ id & Link quality status & Route ID & Energy Status & CRC \\
\hline 2 & 2 & 1 & 4 & 4 \\
\hline
\end{tabular}




\section{Simulation Setup}

The proposed approach is tested using network simulation tool. It is the open-source tool where it is user friendly. The used language for NS2 is the tool command language which is the resemble of $\mathrm{C}++$. The simulation coverage area used for RLRA is $1000 \times 1000 \mathrm{sq} \cdot \mathrm{m}$. The simulation settings are tabulated in Tab. 2. The following parameters are used to validate the performance The following QoS metrics are used to validate the performance of the proposed protocol with existing schemes.

Table 2: Simulator settings for RLRA

\begin{tabular}{ll}
\hline No. of mobile nodes & 150 node \\
\hline Routing protocol & AODV \\
Area size & $1000 \times 1000 \mathrm{sq} \cdot \mathrm{m}$ \\
Mobility model & Random walk \\
Traffic & Constant Bit Rate \\
MAC & IEEE 802.11 \\
Frequency for transmission & $2.4 \mathrm{GHz}$ \\
Data packet rate & 5 packets $/ \mathrm{sec}$ \\
\hline
\end{tabular}

Propagation delay: It is the delay where the packets are propagated from node to node during transmission phase.

Network lifetime: It is the maximum energy after data transmission.

Packet delivery ratio: The ratio which is calculated from packets delivered to packets sent.

Overhead: It means that number of excessive packets present in link where packets may not able to reach sink node.

Energy efficiency: It is defined as the maximum energy obtained to the total available energy.

The proposed protocol RLRA is compared with previous schemes MOALO [10] and BOLSR [11], FTQRP [14].

Fig. 3 illustrates the energy efficiency analysis of RLRA, FTQRP, BOLSR and MOALO. From the results, it is seen that RLRA achieves high energy efficiency than existing schemes due to presence of energy model by consuming less energy for transmission and reception.

Fig. 4 shows the overhead of proposed and existing schemes. The overhead of RLRA is less compared to existing schemes due to stable route discovery and link quality attainment.

Fig. 5 shows the performance of packet delivery ratio of RLRA and existing schemes. The RLRA achieves more packet delivery ratio from the results. It is because of optimal route selection and effective packet forwarding scheme.

Fig. 6 shows the results of propagation delay in $\mathrm{x}$ axis and simulation time in $\mathrm{y}$ axis. While varying the simulation time, the propagation delay of RLRA achieves low value compared to existing schemes.

Fig. 7 shows the results of network lifetime. From the results, RLRA attains more network lifetime due to least energy consumption of packets and optimal route selection. Tab. 3 shows the performance analysis between prosed system and existing system. 


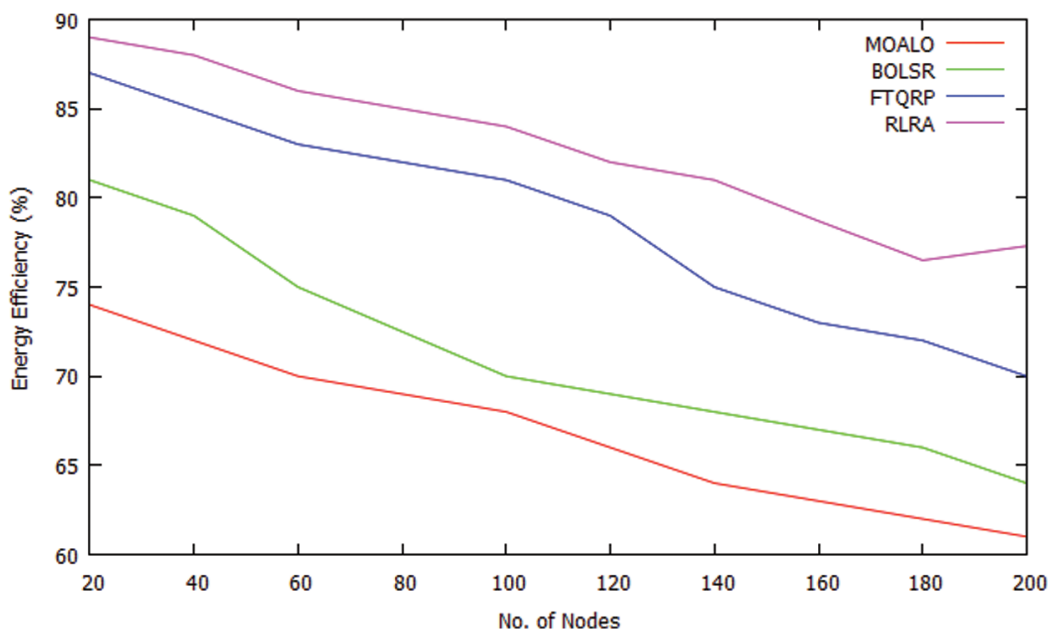

Figure 3: Energy efficiency $v s$. No. of nodes

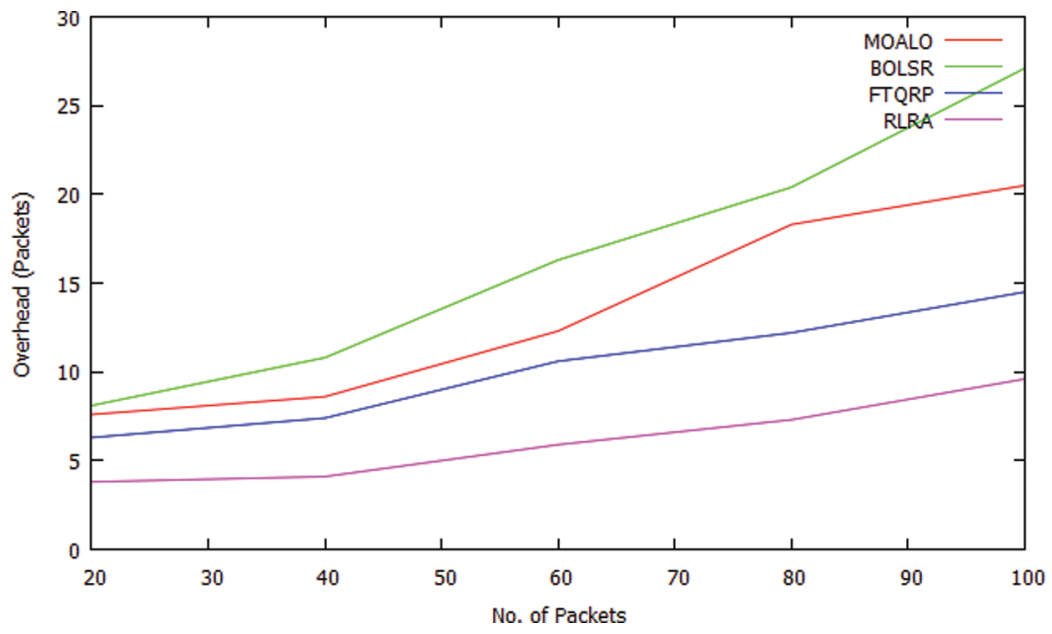

Figure 4: Overhead vs. No. of control packets

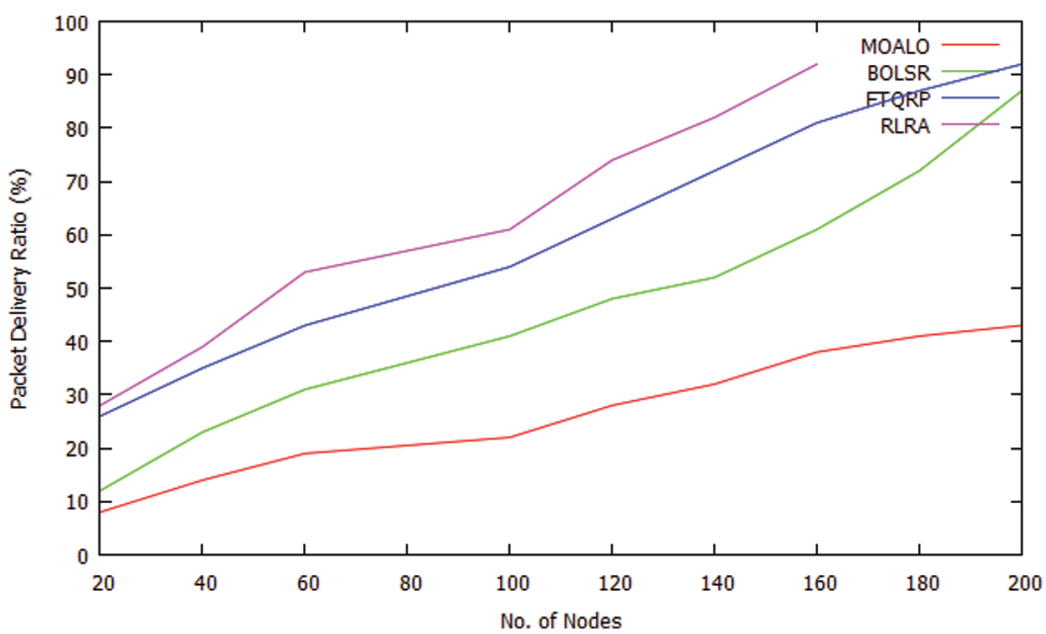

Figure 5: Packet delivery ratio vs. No. of nodes 


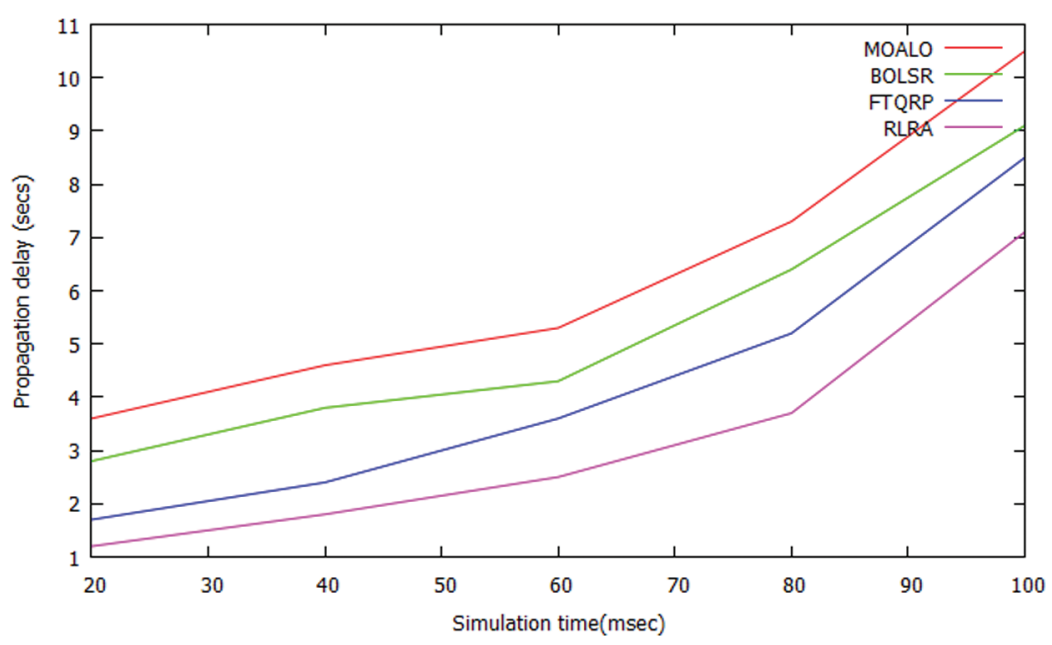

Figure 6: Propagation delay vs. Simulation time

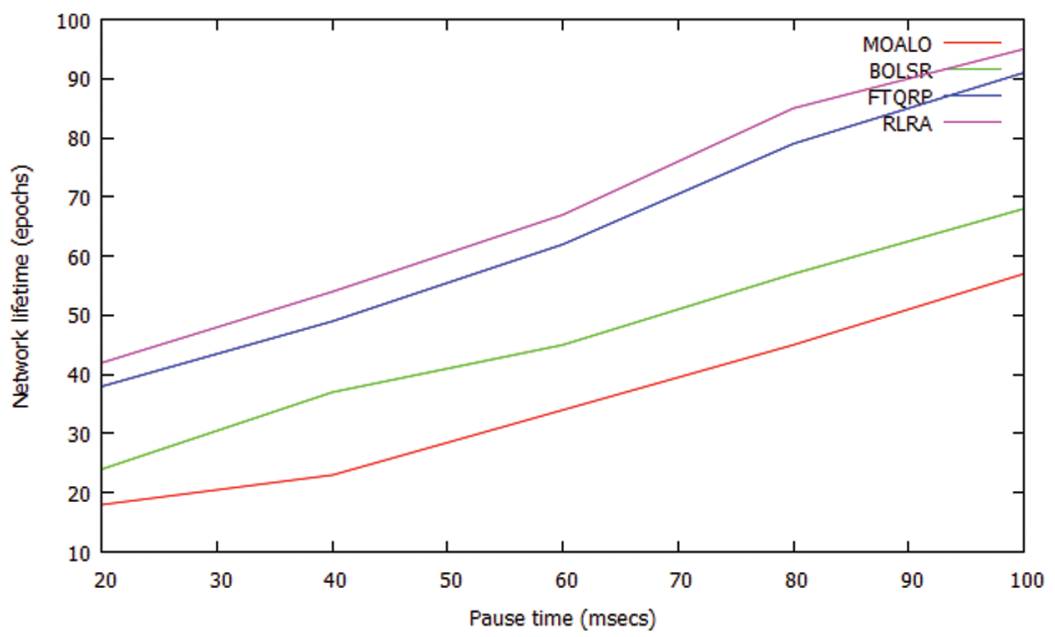

Figure 7: Network lifetime vs. Pause time

Table 3: Performance Analysis

\begin{tabular}{lllll}
\hline $\begin{array}{l}\text { Methods } \\
\text { Parameters }\end{array}$ & MOALO & BOLSR & FTQRP & RLRA \\
\hline Propagation delay (msecs) & $3.6-10.5$ & $2.8-9.1$ & $1.7-8.5$ & $1.2-7.1$ \\
Overhead (Pkts) & $7.6-20.5$ & $8.1-27.1$ & $6.3-14.5$ & $3.8-9.6$ \\
Packet delivery ratio (\%) & $8-38$ & $12-61$ & $26-81$ & $28-92$ \\
Network lifetime (epochs) & $18-57$ & $24-68$ & $38-91$ & $42-95$ \\
Energy efficiency (\%) & $74-63$ & $81-67$ & $87-73$ & $89-79$ \\
\hline
\end{tabular}

From the results, RLRA achieves more packet delivery ratio, more network lifetime and high energy efficiency, less delay and overhead than existing schemes. 


\section{Conclusion}

In ad hoc networks, balancing both link failure and energy will be the major task. in ad hoc networks. In existing schemes, it is failed to balance both metrics. In our proposed model, RLRA is introduced to meet the challenges of ad hoc networks i.e., high energy efficiency and more network lifetime. Network model is introduced to decide the level of reliability and fault tolerant. Multicast routing is established from source to sink node Based on path reliability. The estimation of link quality is done Based on epoch size and length and the optimal multicast routes are discovered from cluster head to cluster member. Energy efficient model is integrated in the network to obtain total energy and save the residual energy. From the results, RLRA achieves more packet delivery ratio, more network lifetime and high energy efficiency, less delay and overhead than existing schemes.

Funding Statement: The authors extend their appreciation to the Deanship of Scientific Research at King Khalid University, Kingdom of Saudi Arabia for funding this workthrough General Research Project Under the grant number (RGP.1/262/42).

Conflicts of Interest: The authors declare that they have no conflicts of interest to report regarding the present study.

\section{References}

[1] K. VinothKumar, T. Jayasankar, M. Prabhakaran and V. Srinivasan, "Fuzzy logic based efficient multipath routing for mobile adhoc networks," Applied Mathematics \& Information Sciences, vol. 11, no. 2, pp. 449455, 2017.

[2] W. K. Kuo and S. H. Chu, "Energy efficiency optimization for mobile ad hoc networks," IEEE Access, vol. 4, pp. 928-940, 2016.

[3] H. Riasudheen, K. Selvamani, S. Mukherjee and I. R. Divyasree, "An efficient energy-aware routing scheme for cloud-assisted MANETs in 5G," Ad Hoc Networks, vol. 97, pp. 102-121, 2019.

[4] L. Zhang and Y. Wang, "An offline roadside unit on-off scheduling algorithm for energy efficiency of ad hoc networks," IEEE Access, vol. 6, no. 26, pp. 59742-59751, 2018.

[5] Y. Peng, F. Al-Hazemi, H. Kim and C. Youn, "Design and optimization for energy-efficient cooperative mimo transmission in ad hoc networks," IEEE Transactions on Vehicular Technology, vol. 66, no. 1, pp. 710-719, 2016.

[6] K. R. Malekshan and W. Z. Huang, "Joint scheduling and transmission power control in wireless ad hoc networks," IEEE Transactions on Wireless Communications, vol. 16, no. 9, pp. 5982-5993, 2017.

[7] V. Tilwari, K. Dimyati, N. Hindia, A. Fattouh and I. Sadegh Amiri, "Mobility, residual energy, and link quality aware multipath routing in manets with q-learning algorithm," MDPI, Applied Science, vol. 9, no. 8, pp. 1-23, 2019.

[8] K. P. K. Rao and T. Senthilmurugan, "Energy efficient clustering technique using k-means and aodv-aco routing with secured AES cryptography in MANET," International Journal of Intelligent Engineering and Systems, vol. 12, no. 3, pp. 292-302, 2019.

[9] J. Ramya and M. Sumathi, "Efficient energy consumption in MANET by AODV," ERR, International Journal for Research in Applied Science \& Engineering Technology, vol. 7, no. 12, pp. 853-860, 2019.

[10] H. Kanagasundaram and K. Ayyaswamy, "Multi objective alo based energy efficient and secure routing OLSR protocol in MANET," International Journal of Intelligent Engineering and Systems, vol. 12, no. 1, pp. 74-83, 2019.

[11] M. Ahmed Jubair, A. Salama, A. Mostafa, R. C. Muniyandi, H. Mahdin et al., "Bat optimized link state routing protocol for energy-aware mobile ad-hoc networks," Sensors, vol. 11, no. 11, pp. 1-22, 2019.

[12] L. N. Fatima, S. H. Mahin and F. Taranum, "Efficient strategies to reduce power consumption in MANETs," Peer Journal of Computer Science, vol. 5, no. 2, pp. 1-23, 2019. 
[13] K. Kaur and K. Kaur, "Recent trends toward fault tolerance techniques in MANET," International Journal of Computer Applications, vol. 120, no. 22, pp. 47-52, 2015.

[14] M. L. Ravichandra and C. Reddy, "Fault tolerant QoS routing protocol for MANETs," in Proc. IEEE Int. Conf. on Advanced Computing, Bhimavaram, India, pp. 623-628, 2016.

[15] S. Palaniappan and K. Chellan, "Energy-efficient stable routing using QoS monitoring agents in MANET," EURASIP Journal on Wireless Communications and Networking, vol. 13, pp. 1-11, 2015.

[16] N. Chaudhary, E. Shiv Kumar Goel and N. Goel, "A deep analysis: Highly robust fault tolerant secure optimized energy ad-hoc networks methodologies for mobile nodes," International Journal of Advanced Research in Computer Science and Software Engineering, vol. 5, no. 7, pp. 540-543, 2015.

[17] F. Tavakoli, M. Kamarei and G. Reza Asgari, "An efficient fault-tolerance routing algorithm for mobile ad-hoc networks," Journal of Advances in Computer Research, vol. 7, no. 2, pp. 23-40, 2016.

[18] S. Nallusamy, S. Appavupillai and S. Ponnusamy, "Mobile agents based reliable and energy efficient routing protocol for MANET," International Journal of Intelligent Engineering and Systems, vol. 9, no. 3, pp. 110116, 2016.

[19] M. Rani and Er. Amit Chhabra, "Modified LA to optimize the performance of mobile ad hoc network," International Journal of Engineering Science and Computing, vol. 10, no. 35, pp. 1283-1286, 2015.

[20] R. Meena, S. Raghuwanshi and Y. Kumar, "Threshold based energy efficient fault tolerance transparent scheme in MANET," International Journal of Advanced Computer Technology, vol. 5, no. 5, pp. 87-93, 2016.

[21] A. E. Narayanan, R. Devi and A. Vincent Jayakumar, "An energy efficient cluster head selection for fault tolerant routing in MANET," International Journal of Engineering and Technology, vol. 5, no. 2, pp. 1281-1289, 2013.

[22] J. Nandhini and D. Sharmila, "Secured message distribution based on multi-bit digital signature in mobile ad-hoc network," International Journal of Advanced Engineering Technology, vol. 8, no. 1, pp. 579-586, 2016.

[23] M. S. Gaur, S. Todi, V. Rao, M. Tripathi and R. Kushwaha, "Multi-constraints link stable multicast routing protocol in MANETs," Ad Hoc Networks, vol. 63, no. 2, pp. 115-128, 2017. 